\title{
Caso 06/2005 - Insuficiência Cardíaca Progressiva em Paciente de 65 Anos de Idade Portador de Marcapasso Cardíaco Artificial
}

\author{
Case 06/2005 - Progressive Heart Failure in a 65 Year-Old Man with Permanent \\ Cardiac Pacemaker
}

Abrão Abuhad, Carlos Alexandre W. Segre, Lea Maria Macruz Ferreira Demarchi, Vera Demarchi Aiello Instituto do Coração do Hospital das Clínicas - FMUSP - São Paulo, SP

Homem de 65 anos de idade procurou atendimento médico em razão de falta de ar e edema.

Há quinze anos apresentou vários episódios de síncope em um mesmo dia. As síncopes não foram precedidas de palpitações e tiveram duração de aproximadamente um minuto. Não procurou atendimento médico na ocasião. Tempos depois, apresentou cansaço desencadeado por esforços habituais e edema de membros inferiores. Procurou avaliação médica e foi informado ser portador de cardiopatia da doença de Chagas.

Há cerca de um ano, apresentou novo episódio de síncope e piora da dispnéia, que passou a ser desencadeada por mínimos esforços, e também repetidos episódios de pré-síncopes.

No dia 26/2/2002 procurou atendimento por nova síncope. Fazia uso de digoxina 0,25 mg diariamente há um mês, a qual havia sido prescrita como tratamento de palpitações paroxísticas.

O exame físico (26/2/2002) revelou paciente orientado, freqüência cardíaca de 22 bpm e pressão arterial 130/70 mmHg. A semiologia pulmonar foi normal. A semiologia cardíaca revelou bulhas arrítmicas, sem sopros. 0 exame do abdome foi normal; não havia edema de membros inferiores.

O eletrocardiograma (26/2/2002) revelou bloqueio atrioventricular total, com escape ventricular, com freqüência cardíaca de 20 bpm e sobrecarga atrial esquerda (fig. 1). 0 teste da atropina (40 $\mu \mathrm{g} / \mathrm{kg}$ ) não desencadeou melhora da condução atrioventricular, embora tenha elevado a freqüência do nó sinusal de 47 bpm para 100 bpm (fig. 2).

Foi submetido a implante de marcapasso cardíaco provisório, no modo VVI, nesse mesmo dia e definitivo no modo DDDR em 26/2/2002. O procedimento transcorreu sem complicações e o paciente recebeu alta no segundo dia de pós-operatório (1/3/2002).

Em consulta ambulatorial, queixou-se de dispnéia aos grandes esforços; o exame físico e o funcionamento do marcapasso estavam normais (11/4/2002).

Houve nova avaliação clínica e do marcapasso (11/11/2002); o paciente não se queixou de sintomas cardiovasculares e o marcapasso estava com funcionamento dentro dos parâmetros esperados. Contudo, pela presença de arritmia atrial o modo de estimulação foi convertido para VVIR. Fazia uso de furosemida $40 \mathrm{mg}$, captopril 25 mg e digitoxina 0,1 mg diários.

Procurou atendimento médico em 1/4/2003 por piora da dispnéia, agora presente mesmo no repouso e no decúbito, além de aparecimento de edema de membros inferiores e de inapetência. A pressão arterial foi 90/60 $\mathrm{mmHg}$; a freqüência cardíaca $75 \mathrm{bpm}$; a ausculta pulmonar revelou estertores crepitantes em bases, a cardíaca revelou bulhas arrítmicas e sopro sistólico $++/ 4+$ em área mitral; o fígado foi palpado a $4 \mathrm{~cm}$ do rebordo costal e havia sinais de ascite, e edema $+++/ 4+$ em membros inferiores.

A medicação em uso era: furosemida $40 \mathrm{mg} / \mathrm{dia}$, mononitrato de isossorbida $60 \mathrm{mg}$, hidralazina $75 \mathrm{mg}$ e amiodarona $200 \mathrm{mg}$ diários, além de warfarina 7,5 mg alternados com 2,5 mg diários.

O eletrocardiograma (1/4/2003) revelou taquicardia atrial com freqüência de $214 \mathrm{bpm}$, com ondas $P$ amplas em $V_{1}$, sugerindo sobrecarga atrial direita, bloqueio atrioventricular total e estimulação ventricular por marcapasso artificial com freqüência de 75 bpm (fig. 3).

Os exames laboratoriais (1/4/2003) revelaram: 14,4 g/dl de hemoglobina, hematócrito 44\%, 8.900 leucócitos/ $\mathrm{mm}^{3}$ (79\% neutrófilos), uréia de $136 \mathrm{~m}$ g/dl, creatinina

Editor da Seção: Alfredo José Mansur (ajmansur@incor.sup.br) Editores Associados: Desidério Favarato (dclfavarato@incor.usp.br) Vera Demarchi Aiello (anpvera@incor.usp.br)

Correspondência: Vera Demarchi Aiello • InCor-Av. Dr. Enéas de Carvalho Aguiar, 44 - 05403-000 - São Paulo, SP 


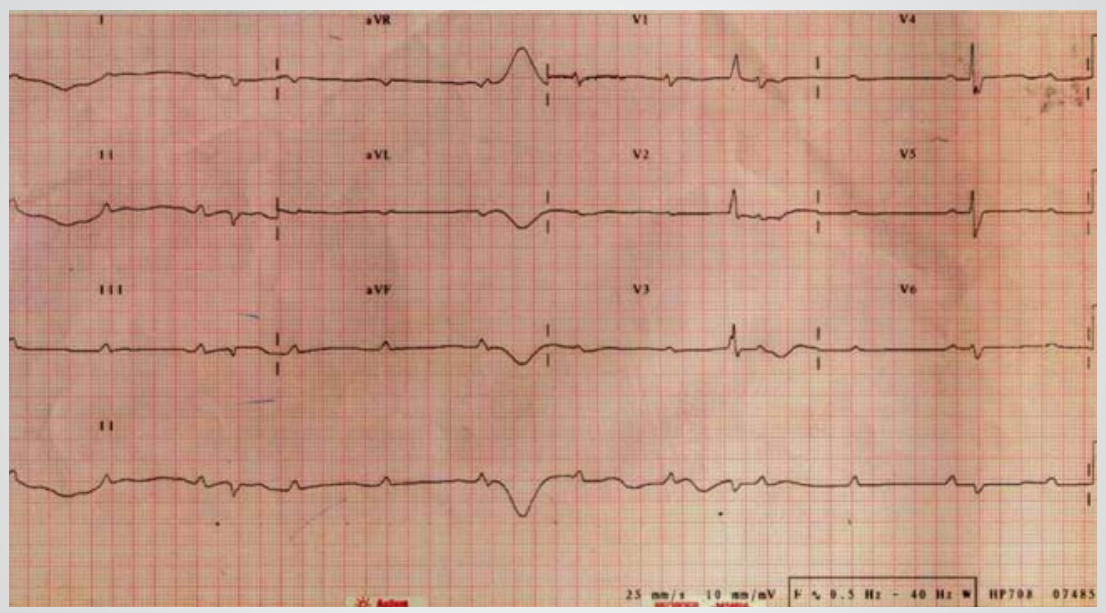

Fig. 1 - ECG - Bloqueio atrioventricular total

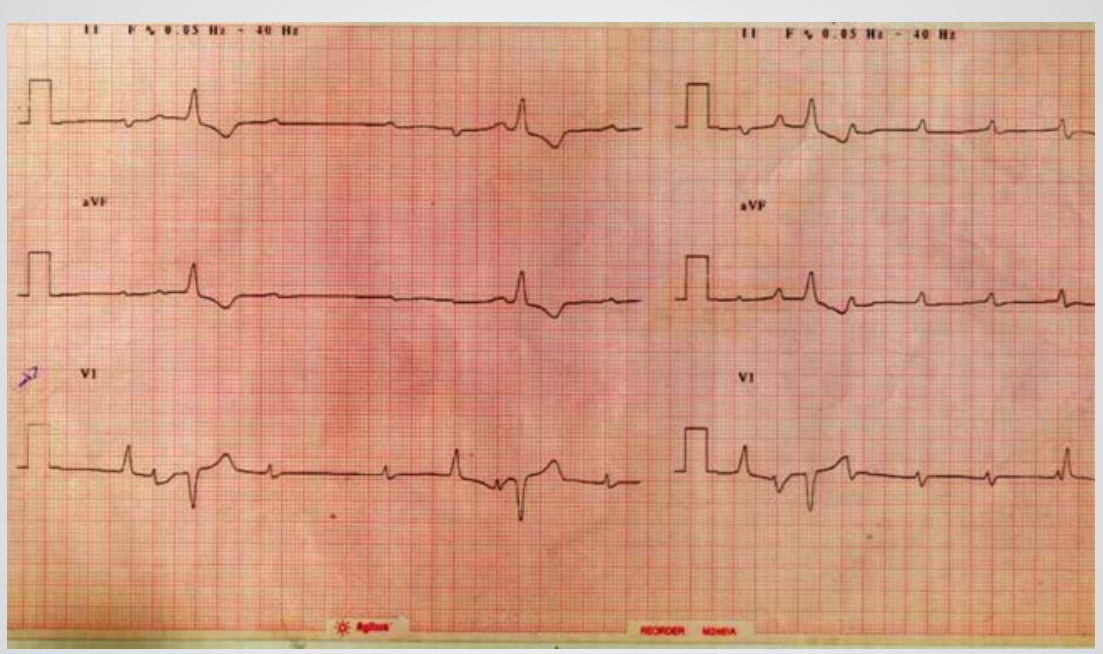

Fig. 2 - ECG - Resposta ao teste com $40 \mu \mathrm{g} / \mathrm{kg}$ de atropina

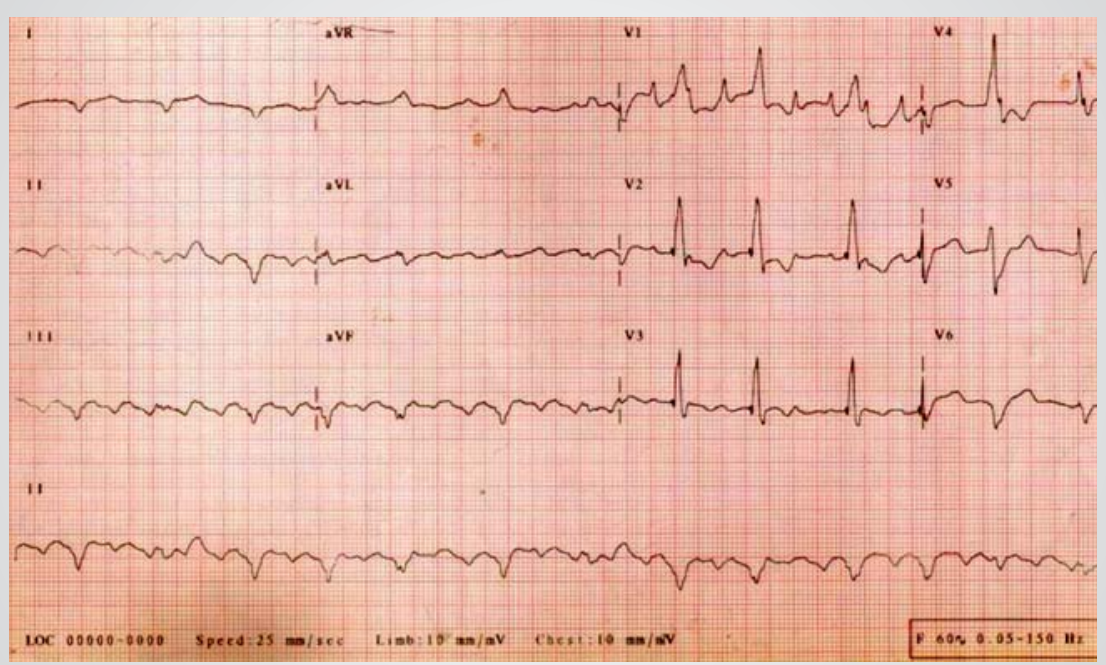

Fig. 3 - ECG - Estimulação com marcapasso artificial 
2,7 mg/dl, $132 \mathrm{mEq} / \mathrm{l}$ de sódio e 4,2 $\mathrm{mEq} / \mathrm{l}$ de potássio. O INR era 17,4, a relação de tempos TTPA 3,41 e a contagem de plaquetas $116.000 / \mathrm{mm}^{3}$. A evolução dos exames laboratoriais é mostrada na tabela I.

Foram feitos os diagnósticos de insuficiência cardíaca descompensada, insuficiência renal e taquiarritmia atrial. Foram prescritos solução de dobutamina, amiodarona 400 mg e enoxaparina 120 mg diários.

O paciente evoluiu com necessidade de uso de agentes vasoativos por via intravenosa e apresentou fraqueza muscular generalizada, com diminuição dos reflexos miotáticos e três dias após apresentou rebaixamento de consciência (madrugada de 10/4/2003), com necessidade de intubação orotraqueal seguida de parada cardíaca nãoresponsiva às manobras de ressuscitação (10/4/2003).

\begin{tabular}{lllll}
\multicolumn{5}{c}{ Tabela I - Evolução laboratorial } \\
\hline Data: & $1 / 4$ & $3 / 4$ & $7 / 4$ & $9 / 4$ \\
\hline Hemoglobina (g/dl) & 14,4 & 12,8 & 13,1 & 15 \\
\hline Hematócrito (\%) & 44 & 35 & 35 & 39 \\
\hline Plaquetas/mm & & 116.000 & 62.000 & 17.000 \\
\hline Leucócitos/mm & 8.900 & 7.300 & 9.100 & 5.100 \\
\hline Bastóes (\%) & 2 & 2 & 11 & 36 \\
\hline Segmentados (\%) & 78 & 67 & 78 & 50 \\
\hline Uréia (mg/dl) & 136 & 115 & 94 & 162 \\
Creatinina (mg/dl) & 2,7 & 2,3 & 1,9 & 3,6 \\
\hline Na (mEq/l) & 132 & 135 & 130 & 129 \\
\hline K (mEq/l) & 4,2 & 3,7 & 4,4 & 6,3 \\
\hline CK (UI/l) & & & & 741 \\
\hline AST (UI/l) & & & & 88 \\
\hline ALT (UI/l) & & & & 18 \\
\hline DHL (UI/l) & & & & 1361 \\
\hline TP (INR) & 3,41 & 3,41 & 1,9 & \\
\hline
\end{tabular}

\section{Aspectos Clínicos}

Trata-se de um paciente de 65 anos com história de cardiopatia chagásica associada a síncopes recorrentes. $\mathrm{Na}$ evolução, uma síndrome de insuficiência cardíaca progressiva agravou sua condição após início de estimulação cardíaca artificial, indicada por bloqueio atrioventricular total. Apresentou piora clínica culminando com colapso circulatório.

Os primeiros sintomas apresentados pelo paciente, então com aproximadamente cinqüenta anos, foram síncopes de repetição. Com relação às sincopes, uma série de diagnósticos diferenciais pode ser formulada, sendo nos pacientes sem cardiopatia estrutural a etiologia vaso-vagal a mais freqüente ${ }^{1}$.

Em nosso meio, a presença de cardiopatia modifica consideravelmente o prognóstico das síncopes, levando a uma mortalidade duas a três vezes maior, sendo um fator de risco independente de morte súbita².

Não existe relato de investigação das síncopes, e alguns meses depois o paciente passou a apresentar dispnéia aos esforços habituais e edema de membros inferiores, sintomas compatíveis com insuficiência cardíaca.
A insuficiência cardíaca, via final das moléstias que acometem o coração, atinge cerca de seis milhões de brasileiros, causando quatrocentas mil internações por ano e alta mortalidade. É a principal causa de internação acima dos sessenta anos ${ }^{3}$.

Na investigação inicial para o quadro de insuficiência cardíaca congestiva, apresentou sorologia positiva para doença de Chagas, não havendo história de hipertensão ou insuficiência coronariana, as outras duas etiologias mais freqüentes para essa síndrome ${ }^{3}$.

A doença de Chagas ainda é causa freqüente de insuficiência cardíaca em nosso meio, constituindo um problema de saúde pública, com cerca de seis milhões de brasileiros infectados pelo protozoário. Estima-se que desses, 20\% a 30\% desenvolverão algum tipo de doença, sendo a cardíaca a mais freqüente ${ }^{4}$.

Esse paciente tem faixa etária de início dos sintomas compatível com a de pacientes que desenvolvem sintomas de insuficiência cardíaca por Chagas.

No início, a única medicação prescrita foi a digoxina, para tentar diminuir a incidência de "palpitações". Alguns autores recomendariam o uso de amiodarona no tratamento de arritmias na doença de Chagas, ao invés da digoxina, pois há evidências que mostram benefício no seu emprego ${ }^{5}$.

Várias publicações mostraram as incidências das diversas formas de arritmias em chagásicos ${ }^{6-8}$ : disfunção do nó sinusal $52 \%$ a $62 \%{ }^{9}$, BAVT 3\% a $27 \%$, e taquicardia ventricular, cuja exata incidência é desconhecida, por ser episódica e de difícil documentação ${ }^{8}$.

Aos 64 anos, caracteriza-se um paciente com miocardiopatia chagásica crônica e síncopes. 0 estudo de Martinelli Filho et al. mostra que no grupo de pacientes chagásicos crônicos a principal causa de síncope são as arritmias ventriculares (43\%), seguida pelos bloqueios atrioventriculares paroxísticos $(21 \%)^{10}$. Para investigação das síncopes poderia ser realizado estudo eletrofisiológico para se comparar os traçados de arritmias induzidas aos dos eventos espontâneos. Porém, na avaliação de síncope inexplicada nenhum traçado eletrocardiográfico no momento do episódio está disponível. A relação entre esses achados e os eventos clínicos será um diagnóstico presuntivo, a menos que o sintoma tenha reprodutibilidade no laboratório. A indução de taquicardia ventricular (TV) monomórfica sustentada, taquicardia supraventricular e bloqueio no sistema His-Purkinje são de valor diagnóstico e prognóstico em pacientes com síncope inexplicada. Em aproximadamente $35 \%$ dos casos com resultado negativo, os pacientes continuam a experimentar episódios recorrentes. Nesses casos, não se descarta uma arritmia como causa dos sintomas; entretanto, a morte súbita parece ser rara nessa situação ${ }^{11}$.

Em 26/2/2002 o paciente apresentou novo episódio de síncope, sendo documentado um bloqueio atrioventricular total (BAVT) com freqüência cardíaca de 22 batimentos por minuto. A abordagem clínica dos bloqueios atrioventriculares consiste na avaliação de seu caráter 
reversível, quando desaparece com a eliminação de sua causa (isquemia, agentes depressores do sistema de condução), e irreversível, quando determinado por disfunção definitiva do sistema de condução. No teste com atropina houve aumento na freqüência sinusal, mas o bloqueio atrioventricular total permaneceu, demonstrando alteração intrínseca do sistema de condução e não ação do digital.

Agora temos um paciente com alteração cardíaca estrutural e síncope associada a BAVT. É possível que desde o início do quadro a causa da síncope fosse BAVT intermitente que evoluiu para BAVT permanente. O BAVT é um fator independente de mau prognóstico na cardiopatia chagásica, como mostraram alguns autores ${ }^{4,12}$.

A primeira questão que merece ser discutida é se, neste caso, o BAVT documentado é suficiente para explicar os episódios de síncope ou se haveria indicação de submeter o paciente a estudo eletrofisiológico, pois, como dito antes, na cardiopatia chagásica as taquicardias ventriculares são a causa mais comum de síncope.

As arritmias ventriculares são uma das manifestações mais preocupantes da cardiopatia chagásica crônica, não só por provocarem sintomas, mas principalmente por constituírem importante fator de risco de morte súbita, especialmente se associadas a comprometimento da função ventricular. As manifestações clínicas das arritmias ventriculares podem estar ausentes ou, quando presentes, traduzirem-se por palpitações, pré-síncope, síncope e morte súbita. Assim, num paciente com cardiopatia chagásica, o estudo eletrofisiológico pode ser uma ferramenta importante no diagnóstico etiológico da síncope ${ }^{13}$. Nas diretrizes brasileiras de arritmia, no entanto, não se encontra indicação formal para realização de estudo eletrofisiológico em pacientes nos quais já existe motivo evidente para as síncopes, a causa foi resolvida e não há recorrência. Assim, o estudo eletrofisiológico poderia ser indicado neste paciente com BAVT caso persistissem as síncopes, o que não aconteceu ${ }^{11}$.

O caso teve indicação de implante de marcapasso (MP) definitivo por bloqueio atrioventricular permanente ou intermitente, associado a síncopes e insuficiência cardíaca em março de 2002 ${ }^{12,14,15}$.

Optou-se por estimulação em modo DDD, e não havia descrição de fibrilação atrial (FA) permanente. O paciente evoluiu bem, permanecendo estável, com dispnéia a grandes esforços e marcapasso com funcionamento normal um mês após o implante ${ }^{16}$.

Em novembro de 2002, em consulta ambulatorial, detectou-se uma arritmia atrial, tendo-se optado por mudar o modo de estimulação para VVIR, ainda que o paciente estivesse assintomático. Diversos estudos demonstraram que o modo VVI proporciona altas taxas de complicações, como FA, tromboembolismo e insuficiência cardíaca. Os estudos até o momento mostram que implante de MP em modo DDD, embora não proporcione aumento da sobrevida, melhora a qualidade de vida ${ }^{15}$.
Nessa ocasião, iniciou-se anticoagulação. É possível que a intenção fosse realizar cardioversão com posterior reprogramação do $\mathrm{MP}^{17}$.

O aparecimento de arritmia atrial é fator de mau prognóstico na história natural da cardiopatia chagásica ${ }^{12}$. Existem relatos de que a presença de eletrodo atrial poderia aumentar a incidência de arritmia atrial; no entanto, essa relação não é freqüente e torna-se secundária diante dos benefícios que uma estimulação atrioventricular pode trazer ${ }^{17}$.

No dia $1^{\circ}$ de abril de 2003, esse paciente chega com insuficiência cardíaca descompensada caracterizada por dispnéia ao repouso, edema de membros inferiores, e ascite. Segundo classificação $0^{18}$ que avalia a hemodinâmica clínica, o paciente se encontra no grupo úmido e frio, constituindo o de pior prognóstico. Apresenta critérios de alta gravidade conforme outro método proposto para avaliação de risco: com uréia acima de $45 \mathrm{mg} / \mathrm{dl}$, pressão sistólica na entrada menor que $115 \mathrm{mmHg}$ e creatinina acima de 2,7 mg/dl, chegando a $20 \%$ de mortalidade em trinta dias ${ }^{19}$. Tem ainda um sódio sérico baixo, também critério de mau prognóstico ${ }^{20}$.

Uma série de fatores, isolados ou em conjunto, deve ser considerada como causa de descompensação nesse ponto da evolução, como citaremos a seguir.

Em primeiro lugar, a evolução natural da cardiopatia chagásica; o paciente apresentou piora progressiva com vários marcadores de mau prognóstico: BAVT ${ }^{21}$,

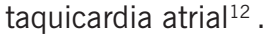

Outro fator seria uma piora da ICC após estimulação cardíaca artificial, levando a uma dessincronização interventricular. Essa provoca disfunção sistólica e diastólica e comprometimento da função mitral, justificando o sopro de insuficiência mitral no exame físico da última internação ${ }^{12}$. Esse detrimento funcional ainda é maior nos miocardiopatas. A perda do sincronismo na contração também dessincroniza o relaxamento, reduzindo o tempo de enchimento ventricular, o que resulta em maior congestão pulmonar e predispõe a $\mathrm{FA}^{22}$.

Já a síndrome do marcapasso pode ser definida como o conjunto de sinais e sintomas presentes no paciente portador de marcapasso com funcionamento normal causados pelo falta de sincronia entre as contrações atriais e ventriculares. Os sintomas são principalmente devidos a diminuição do débito cardíaco e hipotensão. Nesse caso, se levarmos em conta a freqüência de taquicardia atrial, é improvável que a síndrome do marcapasso tenha um papel significativo, uma vez que, nessa freqüência, o período refratário atrial praticamente impede condução retrógrada ${ }^{15,22,23,24}$.

O tromboembolismo pulmonar (TEP) foi achado freqüente em estudo de necropsia realizado no InCor em pacientes com miocardiopatia dilatada, incluindo doença de Chagas, como mostrou Pereira-Barretto com uma incidência por volta de 50\%. Esse autor ressalta a importância de que, mesmo quando diagnosticada, essa complicação não foi descoberta a tempo de ser tratada. 
A dificuldade reside no fato de a embolia não provocar sintomas específicos, mas sim acentuar manifestações comuns aos quadros de insuficiência cardíaca, seja por dispnéia (pela embolia pulmonar) seja por piora da função renal (embolia para esse órgão) ou por desconforto abdominal (embolias viscerais) ${ }^{25}$.

Outro estudo de Arteaga-Fernandez e cols. mostrou uma incidência ainda maior de eventos embólicos pulmonares, especificamente em paciente chagásicos com disfunção miocárdica avançada ${ }^{26}$. Existem ainda relatos de trombose de cabos de marcapasso seguidos de embolia pulmonar ${ }^{27}$.

Existem, portanto, várias evidências a favor do diagnóstico de tromboembolismo pulmonar, sendo ${ }^{28}$ risco aumentado conforme estudos relatados, dados eletrocardiográficos que mostravam nesse momento sinais de sobrecarga atrial direita, e antes o padrão era de sobrecarga atrial esquerda, sugerindo hipertensão pulmonar, que não é comum na evolução natural dessa doença; o aumento da enzima desidrogenase lática e a plaquetopenia. A anticoagulação efetiva não exclui esse diagnóstico ${ }^{29}$.

As infecções devem ser sempre lembradas nas descompensações da insuficiência cardíaca. Nesse caso, no entanto, o paciente só apresentou hemograma compatível com infecção nos dois últimos dias de vida. Não houve febre nem outros dados na história clínica que justificassem um quadro séptico levando a óbito.

A disfunção renal, provavelmente secundária ao baixo débito cardíaco e agravada nesse momento, cursando com hipercalemia tem potencial arritmogênico, possivelmente contribuiu para o desfecho do caso.

(Dr. Abrão Abuhab, Dr. Carlos Alexandre W. Segre)

Hipóteses diagnósticas - Miocardiopatia chagásica, bloqueio atrioventricular total, piora hemodinâmica após implante de marcapasso em modo VVI e piora da insuficiência cardíaca associada a embolia pulmonar.

(Dr. Abrão Abuhab, Dr. Carlos Alexandre W. Segre)

\section{NeCROPSIA}

O exame do coração revelou dilatação das quatro câmaras e afilamento da ponta de ambos os ventrículos, um achado característico da forma crônica cardíaca da doença de Chagas. Havia ainda vegetação comprometendo a face atrial da cúspide anterior da valva tricúspide, em local relacionado à passagem do cabo do marcapasso ventricular (fig. 4 e 5). Havia ainda pericardite fibrinopurulenta e derrame pericárdico tamponante.

Outros achados macroscópicos foram: infartos pulmonares abscedados, bilateralmente, o maior deles medindo $6,0 \times 3,0 \mathrm{~cm}$ nos maiores eixos; dilatação acentuada de ceco e cólon ascendente (megacólon);

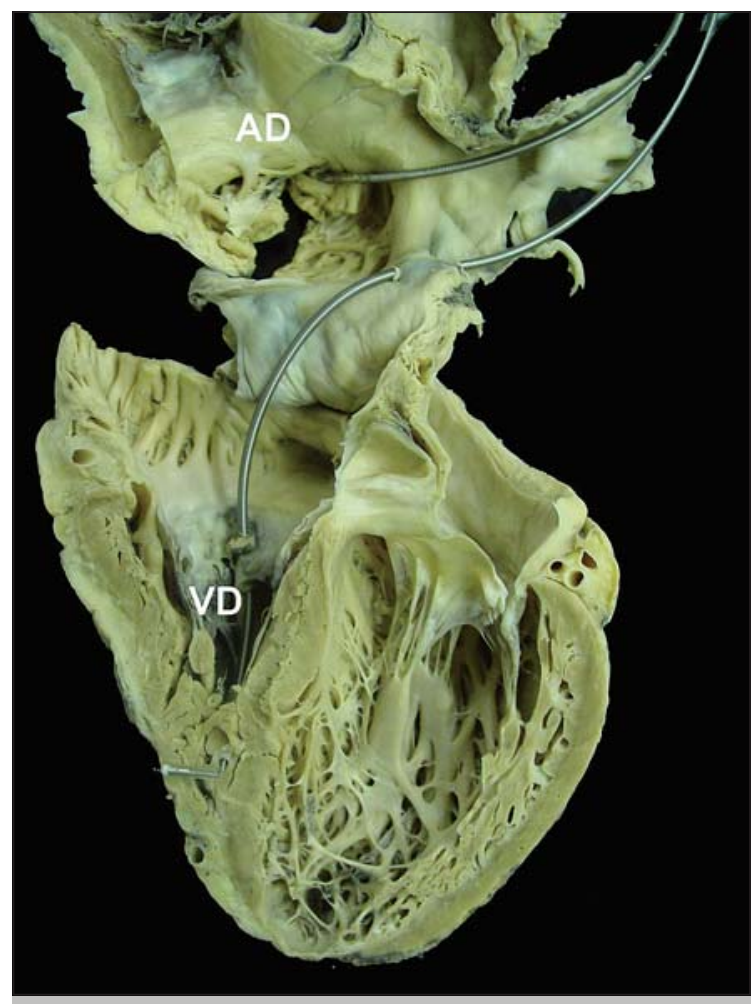

Fig. 4 - Aspecto macroscópico do coração em corte do tipo quatro câmaras, mostrando dois cabos de marcapasso, um deles implantado no átrio direito $(A D)$ e outro no ventrículo direito (VD)

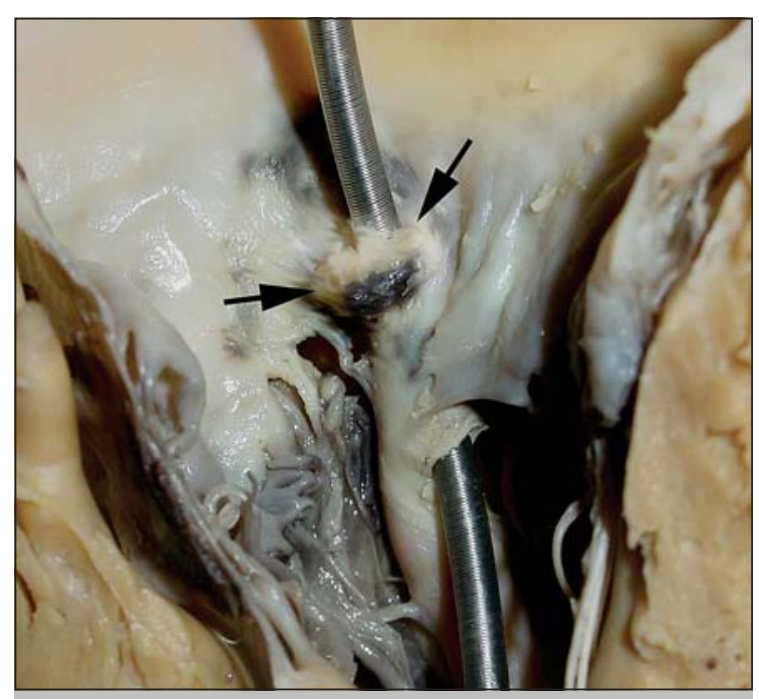

Fig. 5 - Detalhe do marcapasso junto à valva tricúspide, mostrando vegetação de endocardite infecciosa (setas)

edema cerebral.

Microscopicamente detectou-se endocardite bacteriana por cocos Gram-positivos ao redor do eletrodo (fig. 6) e na valva tricúspide. Alguns vasos pulmonares estavam ocluídos por trombos sépticos (fig. 7). O miocárdio mostrou inflamação crônica com moderada atividade acompanhada de fibrose, sem identificação do parasita por meio das colorações histológicas de rotina (fig. 8). 


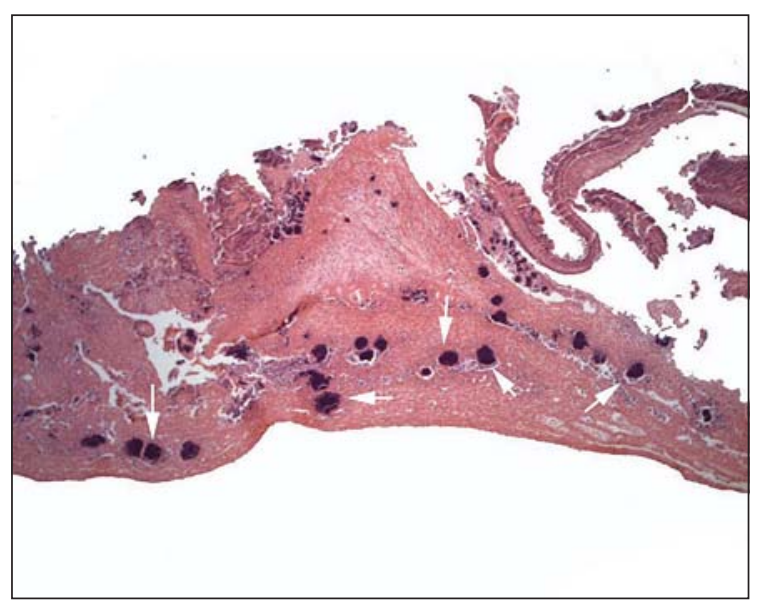

Fig. 6 - Fotomicrografia da vegetação ao redor do marcapasso, mostrando colônias bacterianas (setas) em meio a fibrina. Coloração pela hematoxilina-eosina, objetiva $5 X$

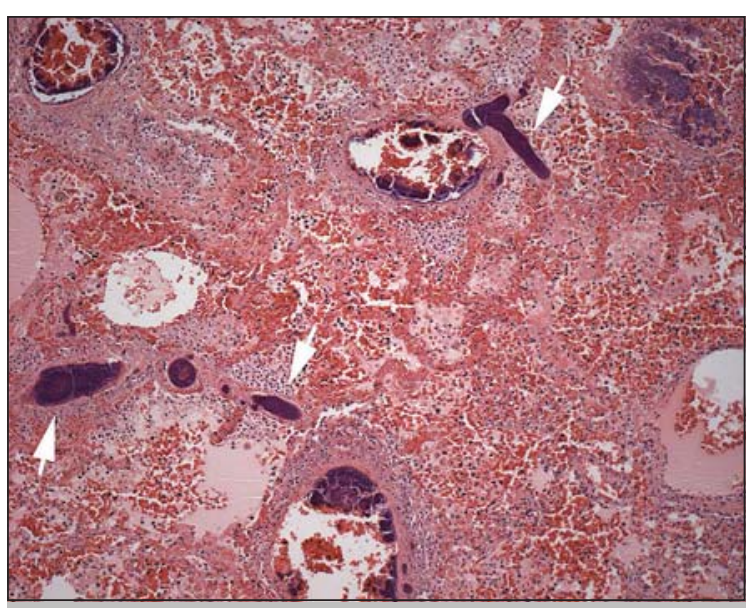

Fig. 7 - Fotomicrografia de tecido pulmonar, exibindo trombos sépticos no interior de arteríolas (setas). Coloração pela hematoxilina-eosina, objetiva $10 \mathrm{X}$

Existiam ainda sinais de choque: dano alveolar difuso pulmonar, necrose hepática centrolobular. A causa do óbito foi choque misto, séptico e cardiogênico.

(Dra. Lea Maria Macruz Ferreira Demarchi, Dra. Vera Demarchi Aiello)

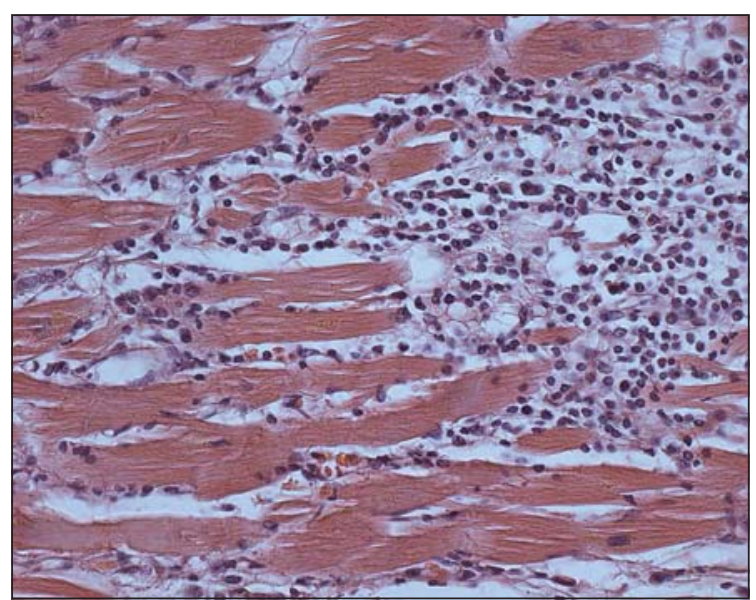

Fig. 8 - Fotomicrografia do miocárdio, mostrando foco de miocardite crônica. Coloração pela hematoxilina-eosina, objetiva $40 X$

\section{Comentários}

Complicações envolvendo implantes de marcapasso compreendem: perfuração de valvas ou paredes miocárdicas (atriais ou ventriculares), trombose, deslocamento, fratura e infecção do eletrodo; e, tardiamente, fibrose ao redor deste, com conseqüente isolamento e perda da condução do estímulo ${ }^{30}$.

A endocardite infecciosa nessa situação pode acometer apenas o cabo do marcapasso, porém mais freqüentemente compromete também estruturas valvares. Os estafilococos aparecem como os organismos causadores mais freqüentes. Em casuística recente de um grupo francês descreveu-se uma incidência de 550 casos/milhão de receptores de marcapasso/ano ${ }^{31}$.

No caso aqui relatado, os abscessos pulmonares são conseqüência de embolização de vegetações de endocardite da valva tricúspide, e a causa de óbito foi choque misto.

(Dra. Vera Demarchi Aiello, Dra. Lea Maria Macruz Ferreira Demarchi)

\section{REFERÊNCIAS}

1. Soteriades ES, Evans JC, Larson MG et al. Incidence and prognosis of syncope. N Engl J Med 2002; 347: 878-85.

2. Kapoor WN. Syncope. N Engl J Med 2000; 343: 1856-62.

3. II Diretrizes da Sociedade Brasileira de Cardiologia para Diagnóstico e Tratamento da Insuficiência Cardíaca. Arq Bras Cardiol 2002; 79 (Supl IV): 3-30.

4. Bernstein M, lanni BM. Seguimento clínico dos portadores de doença de Chagas. Rev Soc Cardiol Estado de São Paulo 2001; 11 (1): 45-54.

5. Doval HC, Nul DR, Grancelli HO, Perrone SV, Bortman GR, Curiel R. Randomised trial of low-dose amiodarone in severe congestive heart failure. Grupo de Estudio de la Sobrevida en la Insuficiencia Cardiaca en Argentina (GESICA). Lancet. 1994; 344: 493-8.

6. Lorga AM, Garzon SAC, Lorga Filho AM. Síncope e cardiopatia chagásica crônica. Rev Soc Cardiol Estado de São Paulo 1999; 9: 191-7.

7. Rofeld A, Fernandes MA, de Barros Camargo N et al. Electrocardiogram in subjects with positive Guerreiro-Machado reaction. Arq Bras Cardiol 1978; 31: 191-4.

8. Martinelli Filho M. Cardiopatia Chagásica Crônica e Síncope. Aspectos clínicos e eletrofisiológicos. São Paulo, 1992. Tese (Doutorado) - Faculdade de Medicina, Universidade de São Paulo. 
9. Benchimol CB, Kreuzig R, Ginefra P, Schlesinger P. A disfunção do nó sinusal na cardiopatia chagásica crônica. Arq Bras Cardiol 1977; 30: 337-44.

10. Martinelli Filho M, Sosa E, Nishioka S, Scanavacca M, Bellotti G, Pileggi F. Clinical and electrophysiologic features of syncope in chronic chagasic heart disease. J Cardiovasc Electrophysiol 1994; 5: 563-70.

11. Diretrizes para Avaliação e Tratamento de Pacientes com Arritmias Cardíacas. Arq Bras Cardiol 2002; 79 (Supl.V): 2-50.

12. Rassi Jr A, Rassi SG, Rassi AG. Sudden Death in Chagas' Disease. 2nd Virtual Congress of Cardiology - http://www.fac.org.ar/scvc/scvirpor/ area por/arrit por.htm

13. Martinelli Filho M, De Siqueira SF, Moreira $\mathrm{H}$ et al. Probability of occurrence of life-threatening ventricular arrhythmias in Chagas' disease versus non-Chagas' disease. Pacing Clin Electrophysiol. 2000; 23: 1944-6.

14. Felker GM, Adams KF Jr, Konstam MA, O'Connor CM, Gheorghiade M. The problem of decompensated heart failure: nomenclature, classification, and risk stratification. Am Heart J. 2003; 145: S18-25.

15. Mitrani RD, Simmons JD, Interian A Jr, Castellanos A, Myerburg RJ. Cardiac pacemakers: current and future status. Curr Probl Cardiol. 1999 Jun;24(6):341-420.

16. Greco OT, Melo CS. Marcapasso na doença de Chagas. Rev Soc Estado de São Paulo 2004; 2: 357-70.

17. Martinelli M, de Arruda Melo S, Nishioka SA et al. Atrioventricular pacemaker. Incidence and causes of reprogramming in long-term follow-up. Arq Bras Cardiol 2001; 76: 7-14.

18. Nohria A, Lewis E, Stevenson LW. Medical management of advanced heart failure. JAMA 2002; 287: 628-40.

19. Fonarow G, Adams KF, Abraham WT, Yancy CW, Boscardin WJ. Risk stratification for in-hospital mortality in acutely decompensated heart failure classification and regression tree analysis. JAMA 2005; 293: $572-80$.

20. Freitas HFG, Chizzola PR, Markus MRP, Bocchi EA, Mansur AJ. Prognóstico em portadores de insuficiência cardíaca sintomática grave. In: Bocchi EA, Pereira Barretto AC. Insuficiência Cardíaca. ( $1^{\text {aed }}$ d) São Paulo: Libbs; 2003.

21. Rassi A, Rassi Jr A, Faria GHDC et al. História natural do bloqueio atrioventricular total de etiologia chagásica. Arq Bras Cardiol 1992; 59 (Supl II): 191.

22. Pachon Mateos JC, Pachon Mateos El, Albornoz RN, Gimenez VM, Pachon Mateos JC. Insuficiência cardíaca - Marcapasso como opção terapêutica. Rev Soc Estado de São Paulo 2000 10: 119-29.

23. Josephson ME. Electrophysical Investigation: General Concepts. In: Josephson ME. Clinic Eletrophysiology. 3rd ed. Lippincott: Williams\&Wilkins; 2002.

24. Barold SS. The Fourth Decade of Cardiac Pacing. Hemodynamic, Eletrophysiological and Clinical Considerations in selection of the Optimal Pacemaker. Chap 119. In: Zipes D. Cardiac Eletrophysiology. 2nd ed. Philadelphia: WB Sauders; 1994.

25. Pereira-Barretto AC. Tromboembolismo na insufciencia cardiaca. Rev Soc Cardiol Estado de São Paulo 2000; 10 (1): 23-31.

26. Arteaga-Fernandez E, Barretto AC, lanni BM et al. Cardiac thrombosis and embolism in patients having died of chronic Chagas cardiopathy. Arq Bras Cardiol 1989; 52: 189-92.

27. Medeiros F, Benvenuti LA, Costa R. A sixty-four-year-old woman with Chagas' disease, who underwent implantation of a permanent cardiac pacemaker and evolved with rapidly progressive heart failure. Arq Bras Cardiol 2001; 77: 167-82.

28. Goldhaber ZS. Pulmonay Embolism. Chap. 52. In: Braunwald E.. Heart Disease. Textbook of Cardiovascular Medicine. 7th ed. Philadelphia: WB Saunders; 2004.

29. Kearon C, Ginsberg JS, Kovacs MJ et al. Extended Low-Intensity Anticoagulation for Thrombo-Embolism Investigators. Comparison of low-intensity warfarin therapy with conventional-intensity warfarin therapy for long-term prevention of recurrent venous thromboembolism. N Engl J Med 2003; 349: 631-9.

30. Schoen FJ, Edwards WD. Pathology of cardiovascular interventions, including endovascular therapies, revascularization, vascular replacement, cardiac assist/replacement, arrhythmia control, and repaired congenital heart disease. In: Silver MD, Gotlieb A, Schoen FJ, eds. Cardiovascular Pathology. 3rd Ed. New York: Churchill Livingstone; 2001: 678-721.

31. Duval X, Selton-Suty C, Alla F et al. Association pour l'Etude et la Prevention de l'Endocardite Infectieuse. Endocarditis in patients with a permanent pacemaker: a 1-year epidemiological survey on infective endocarditis due to valvular and/or pacemaker infection. Clin Infect Dis 2004 ; 39 : 68-74. 